\title{
6
}

\section{Free and forced thermocline oscillations in} Lake Tanganyika

\author{
Olivier Gourgue, Eric Deleersnijder, Vincent Legat \\ Université catholique de Louvain \\ Centre for Systems Engineering and Applied Mechanics (CESAME) \\ 4 Avenue Georges Lemaître, B-1348 Louvain-la-Neuve, Belgium
}

Emmanuel Marchal

$N$-Side s.a.

6 Chemin du Cyclotron. B-1348 Louvain-la-Neuve, Belgium

Laurent White

Princeton University

Geophysical Fluid Dynamics Laboratory

201 Forrestal Road, Princeton, NJ 08536, USA

\subsection{Introduction}

Lake Tanganyika is located on the east of central Africa, and is shared by four developing countries: Democratic Republic of the Congo, Burundi, Tanzania and Zambia. It lies between $3^{\circ} 20^{\prime}$ to $8^{\circ} 45^{\prime} \mathrm{S}$ and $29^{\circ} 05^{\prime}$ to $31^{\circ} 15^{\prime}$ E. It is about $650 \mathrm{~km}$ long and $50 \mathrm{~km}$ wide on average. The mean depth of the lake is about $570 \mathrm{~m}$, with a maximum depth of $1470 \mathrm{~m}$ (Figure 6.1). That makes it the second deepest lake in the world, the deepest being Lake Baikal in Russia. Thermal stratification is well marked and present all year long, so that one can identify two distinct layers: the surface and the bottom layers. The surface layer is composed of relatively warm water $\left(24-28^{\circ} \mathrm{C}\right)$ while the bottom layer is composed of cooler water $\left(\approx 23.5^{\circ} \mathrm{C}\right)$. These two layers are separated by a thermocline, which is a thin layer where the temperature gradient is maximum. The mean depth of the thermocline is about $50 \mathrm{~m}$. As the very deep water almost never reaches the surface, the lake can be categorized as meromictic [Coulter and Spigel, 1991].

This study only focuses on the hydrodynamics. However, it must be kept in mind that the latter exerts a profound influence on a number of chemical [Plisnier et al., 1999] and biological [Descy et al., 2005] properties throughout the lake. It also governs upwelling of nutrients that is essential 


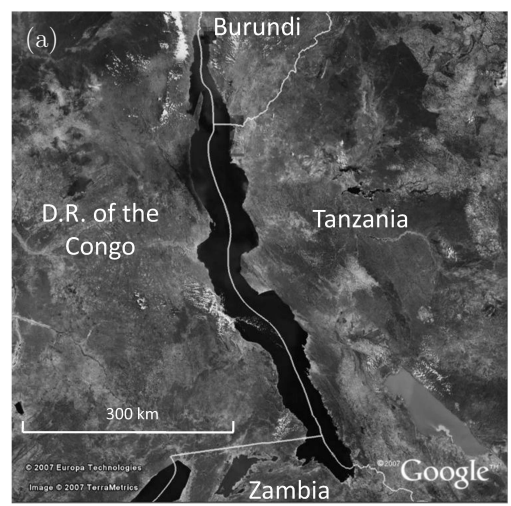

(b)

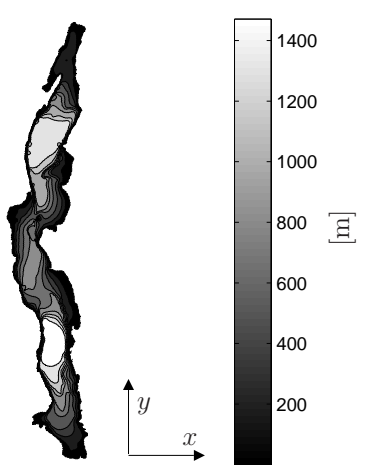

Fig. 6.1. (a) Map of Lake Tanganyika (from Google Earth ${ }^{\mathrm{TM}}$ mapping service), indicating the four neighboring countries. (b) Bathymetry of Lake Tanganyika (with depths measured in metres) and $x$ - and $y$-axis of the Cartesian coordinate system used in the present work.

in maintaining the pelagic food web [O'Reilly et al., 2003]. Water motions are chiefly forced by the surface wind stress. The region undergoes two seasons: the dry season (approximately from May to August), characterized by strong winds mainly blowing northwestward along the main axis of the lake, and the wet season (approximately from September to April), during which the winds are generally weaker.

During the dry season, the wind pushes the warmer surface water toward the northern end of the lake. The thermocline moves upward to compensate for the loss of water at the south and the warmer water accumulated at the north is pulled downward by gravity. The thermocline is then tilted downward toward the north and occasionally outcrops in the southern part of the lake. At the end of the dry season, when the southeasterly winds stop, the surface and bottom layers slide over each other, and the thermocline oscillates to reach a new equilibrium. These waves are reflected at the lake boundaries and gradually transform into standing wave patterns, called internal seiches. By December, the thermocline reaches its mean level (around $50 \mathrm{~m}$ ) but keeps oscillating until the beginning of the next dry season and the onset of the southeasterly winds. The period of these oscillations is about three to four weeks. Indeed, a fundamental oscillation mode of 25 to 30 days has been found both from field data [Coulter and Spigel, 1991] and from a simple model [Mortimer, 1974], and a fundamental mode of about 24 days has been found from a reduced-gravity model with a seasonal wind stress 
forcing [Naithani et al., 2003]. The presence of rather small internal Kelvin waves in the lake has been emphasized by [Naithani and Deleersnijder, 2004] and [Antenucci, 2005].

Herein two types of oscillations are identified: the free and the forced ones. The free oscillations are related to the seasonal cycle of the surface wind stress. When a seasonal wind stress is applied over the whole lake (i.e. a constant wind stress during the dry season and zero wind stress during the wet season, see Figure $6.2 \mathrm{~b}$ ), the thermocline oscillates about two equilibrium states: a tilted thermocline (downward toward the north) during the dry season and a horizontal one during the wet season [Naithani et al., 2003]. The free oscillations are not a direct response to the seasonal wind stress. They are due to the alternation between two different equilibrium states along the year. Therefore, free oscillations can be regarded as triggered by the seasonal wind stress. The period of the first mode of these free oscillations (which is the only significant mode) is about three to four weeks [Naithani et al., 2003].

The forced oscillations are due to the intraseasonal variability of the surface wind stress, which is defined as the difference between the actual wind stress (Figure 6.2a) and the seasonal wind stress (Figure 6.2b). By means of a wavelet analysis, it has been shown that, in the region of Lake Tanganyika, this intraseasonal variability of the surface forcing has a period of three to four weeks [Naithani et al., 2002]. This period is due to the eastward-propagating low-frequency large-scale convection and circulation cells, which get their energy from the seasonally migrating intertropical convergence zone [Madden and Julian, 1971, Madden and Julian, 1994], but also to cloud-radiation [Krishnamurti and Bhalme, 1976] and evaporationwind [Neelin et al., 1987, Lin et al., 2000] feedback processes, and to the interactions between moist convective and dynamical processes [Goswami and Shukla, 1984]. The timescale associated with the intraseasonal variations of the wind forcing is of the same order of magnitude as the period of the free oscillations. Therefore, the oscillations directly forced by the intraseasonal oscillations of the wind stress tend to have a large amplitude, as was seen by [Naithani et al., 2002] who identified this near-resonance phenomenon.

Free [Naithani et al., 2003] and forced [Naithani et al., 2002] thermocline oscillations in Lake Tanganyika have already been studied separately. However, so far, the relative importance of these two types of response and the interactions between them have not been investigated. Doing so by means of the factor separation method is the objective of the present chapter. The wind stress is split into a seasonal component and an intraseasonal one. This leads to a decomposition of the thermocline response into a seasonal and an 

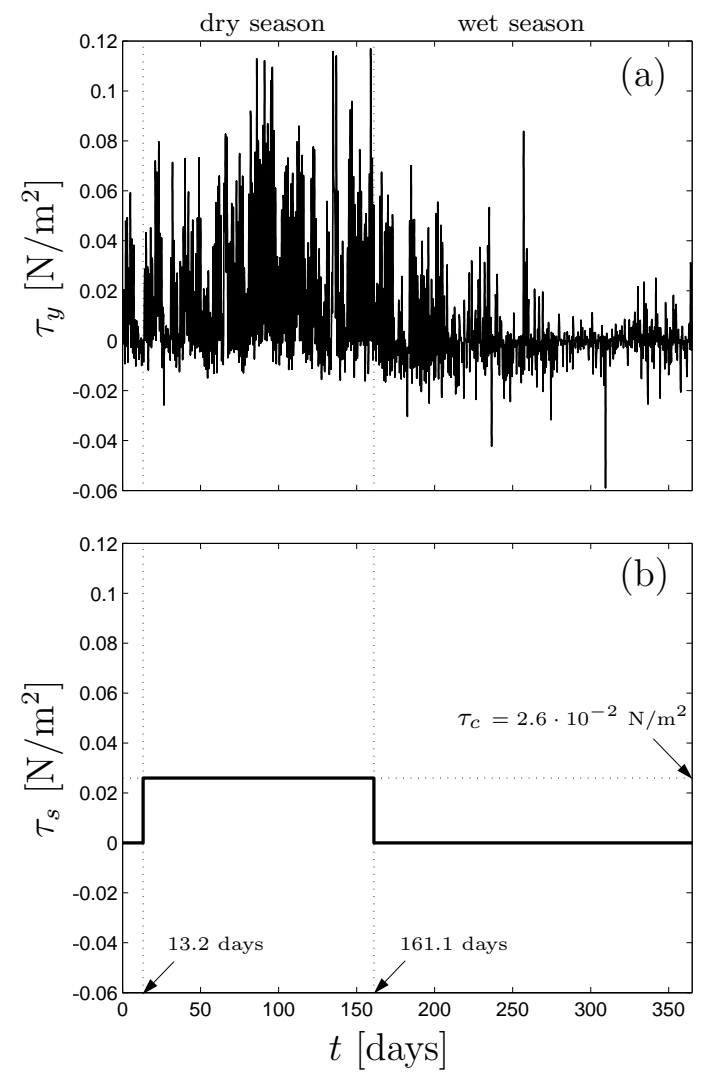

Fig. 6.2. (a) Wind stress in the along-lake direction, measured at Mpulungu in Zambia, every six hours from 1st April 1993 to 31st March 1994, during the FAO/FINNIDA project "Research for the Management of the Fisheries on Lake Tanganyika" GCP/RAF/271/FIN. (b) Seasonal wind stress, with a constant wind stress $\tau_{c}$ during the dry season and zero wind stress during the wet season.

intraseasonal response (respectively responsible for the free and forced oscillations) and also a synergistic term, due to the non-linearity of the problem [Stein and Alpert, 1993]. Therefore, the factor separation method can help in assessing the relative magnitude of the free and forced oscillations of the thermocline in Lake Tanganyika.

\subsection{Model description}

Two-layer models have often been used to simulate wind-induced internal waves in large, stratified lakes. For example, we may cite studies of Lake Biwa in Japan [Kanari, 1975], Lake Ontario in Canada [Schwab, 1977], or 
Lake Kinneret in Israel [Antenucci and Imberger, 2001]. The equations of such models are well known, and references on their design may easily be found in the literature [Csanady, 1967, Kielmann and Simons, 1984]. With two-layer models, there are six variables, i.e. the displacement of the free surface, the displacement of the interface between the two layers, the horizontal velocity components averaged over the surface layer thickness and the horizontal velocity components averaged over the bottom layer thickness.

When the bottom layer is much thicker than the surface one, relevant simplifications may lead to the reduced-gravity model [Naithani et al., 2003]. The reduced-gravity model is a 1.5-layer model in that it focuses on the dynamics of the surface layer, without disregarding completely the bottom one. It has only three variables, i.e. the displacement of the interface between the two layers and the horizontal velocity components averaged over the surface layer thickness. The bathymetry is no longer explicitely taken into account.

Several papers have already shown that a reduced-gravity model is able to simulate rather well the thermocline oscillations in Lake Tanganyika [Naithani et al., 2002, Naithani et al., 2003] since stratification is present all year round and the surface layer is much shallower than the bottom one, i.e. the thickness of the former is about $10 \%$ of that of the latter [Coulter and Spigel, 1991].

The equations of the reduced-gravity model read:

$$
\begin{aligned}
\frac{\partial \xi}{\partial t}+\nabla \cdot(H \boldsymbol{u})= & 0 \\
\frac{\partial}{\partial t}(H \boldsymbol{u})+\boldsymbol{\nabla} \cdot(H \boldsymbol{u} \boldsymbol{u})= & -f \boldsymbol{e}_{\boldsymbol{z}} \times(H \boldsymbol{u})-(\epsilon g) H(\boldsymbol{\nabla} \xi) \\
& +\boldsymbol{\nabla} \cdot(H \nu(\boldsymbol{\nabla} \boldsymbol{u}))+\frac{\boldsymbol{\tau}}{\rho},
\end{aligned}
$$

where $t$ denotes the time and $\boldsymbol{\nabla}$ is the horizontal spatial derivative vector operator; $\xi$ is the downward displacement of the thermocline (Figure 6.3 ), while $\boldsymbol{u}$ is the horizontal velocity vector averaged over the surface layer thickness $H=h+\xi$ (since the displacement of the free surface is neglected), $h$ being the surface layer thicknesses at rest (Figure 6.3); $f$ is the Coriolis parameter; $(\epsilon g)$ is the reduced gravity, where $g$ is the gravitational acceleration and $\epsilon=\frac{\rho_{b}-\rho}{\rho_{b}}$ is the relative density difference (here equal to $6.3 \times 10^{-4}$ in order to be consistent with former studies); $\nu$ is the horizontal eddy viscosity; $\boldsymbol{\tau}$ is the surface wind stress vector; $\rho$ and $\rho_{b}$ are the constant water densities in the surface layer an the bottom layer, respectively.

The standard reduced-gravity model rests on the assumptions that the thermocline is impermeable, implying that the volume of the surface layer 


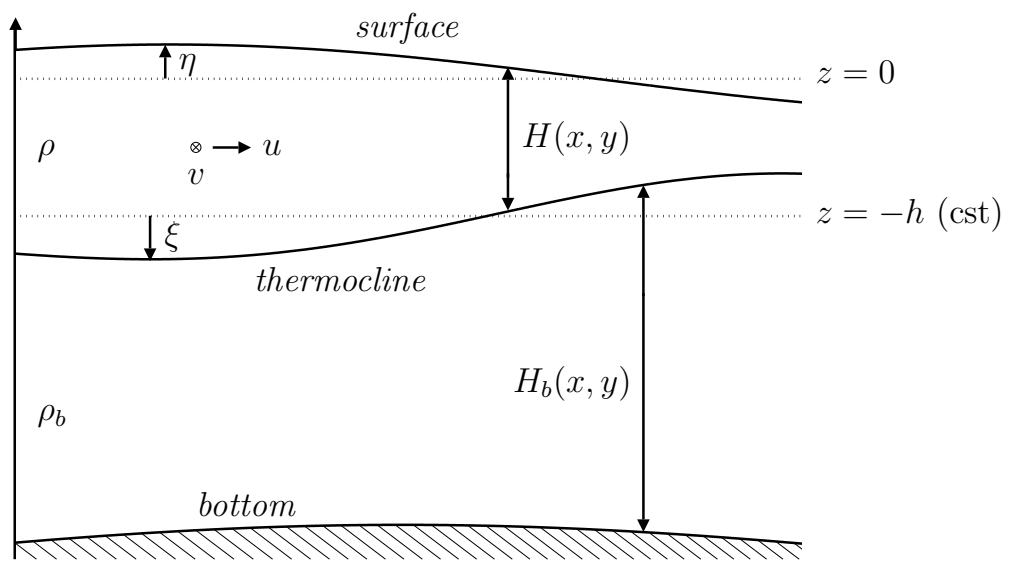

Fig. 6.3. The parameters and variables of a reduced-gravity model: $\eta$ is the upward displacement of the free surface, $\xi$ is the downward displacement of the thermocline; $\rho$ and $\rho_{b}$ are the densities of the surface and bottom layers, respectively; $u$ and $v$ are the components of $\boldsymbol{u}$, the velocity vector averaged over the surface layer; $h$ is the surface layer thickness at rest; $H$ and $H_{b}$ are the instantaneous surface and bottom layer thicknesses, respectively.

remains constant. However, some modifications allow to take into account the water exchanges between the surface and bottom layers, and those due to precipitation, evaporation and rivers [Gourgue et al., 2007]. This approach is not resorted to herein, in order to follow the assumptions of the previous studies [Naithani et al., 2002, Naithani et al., 2003], and because it is not essential for studying the internal oscillations in the lake.

Since the rivers are not taken into account, the lateral boundary of the lake can be assumed to be impermeable. Moreover, we make the assumption of a free slip lateral boundary. The corresponding boundary conditions therefore read:

$$
\begin{aligned}
u_{n} & =0 \\
\frac{\partial u_{t}}{\partial n} & =0,
\end{aligned}
$$

where $u_{n}$ and $u_{t}$ denote the horizontal velocity components normal and tangential to the boundary, respectively, and $\frac{\partial}{\partial n}$ the spatial derivative in the direction normal to the boundary.

To simulate the oscillations of the thermocline, we discretize equations (6.1) and (6.2) on the unstructured mesh displayed in Figure 6.4, using the two-dimensional component of the finite element model SLIM (Second- 


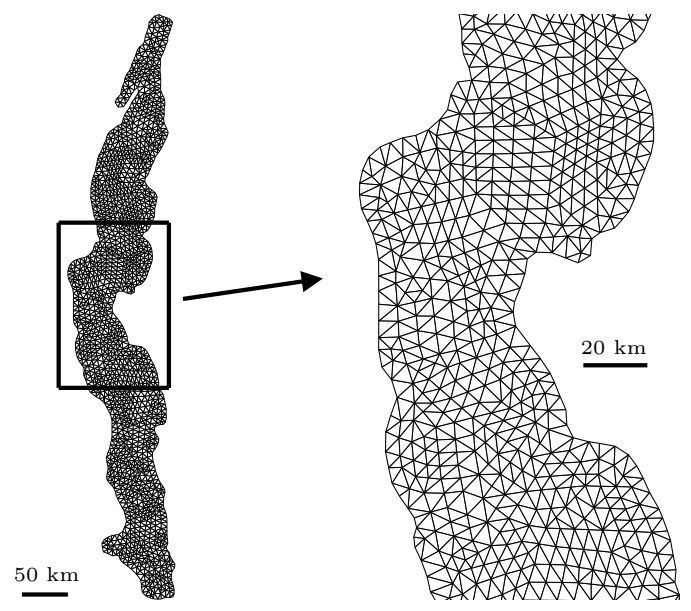

Fig. 6.4. Unstructured mesh of Lake Tanganyika used for the present simulations. There are 2997 triangular elements, whose mean size is about $5 \mathrm{~km}$.

generation Louvain-la-Neuve Ice-ocean Model $\dagger$ ). The elevation and velocity variables are approximated by linear conforming $\left(P_{1}\right)$ and linear nonconforming $\left(P_{1}^{N C}\right)$ shape functions, respectively. Therefore, the elevation nodes are situated at the vertices of each triangle of the mesh, and the velocity nodes at the middle of their edges [Hanert et al., 2005].

Occasionally, the upward displacement of the thermocline can be equal to the reference thickness of the surface layer. To prevent the thermocline from outcropping, a wetting-drying algorithm is implemented. When the water column depth of a node reaches a critical value, which is taken to be $5 \mathrm{~m}$ in this application, the node is considered to be dry. Otherwise, it is a wet node. The wetting-drying algorithm consists in setting to zero the fluxes across the edges of a patch made up of all the triangles sharing a dry node. At each time step, we first compute the elevation field $\xi$. If there are dry nodes, we modify the velocity fields $u$ and $v$ (of the previous time step) with the wetting-drying algorithm, before recomputing the elevation field. This technique is used recursively until there are no more dry nodes. Then, we compute simultaneously the velocity fields $u$ and $v$. This kind of approach was initially introduced for the finite difference method [Balzano, 1998]. It has already been used in a finite difference model of Lake Tanganyika [Naithani et al., 2002, Naithani et al., 2003], and it has been adapted herein to our finite element model [Marchal, 2005].

The initial conditions need no discussion: the numerical results are ana- 
lyzed several years after the beginning of the simulation, i.e. at a time when a periodic regime is reached that is independent of the initial values of the variables.

\subsection{Wind stress forcing decomposition}

Surface wind measurements were made from 1st April 1993 to 31th March 1994 at Mpulungu by the FAO/FINNIDA project "Research for the Management of the Fisheries on Lake Tanganyika" GCP/RAF/271/FIN. Mpulungu is situated at the south end of the lake in Zambia. Following former studies, we assume that this pointwise wind stress may be applied to the entire lake surface [Naithani et al., 2002]. Therefore, the surface wind stress vector $\boldsymbol{\tau}(t)$ is a function of time only. Its components are $\tau_{x}(t)$ and $\tau_{y}(t)$, following the $x$ and $y$ axis of Figure 6.1b, respectively (the $y$-axis is in the along-lake direction, from south to north). The component $\tau_{x}(t)$ of the surface forcing may be neglected, for it is much smaller than $\tau_{y}(t)$, and because of the narrowness of the lake [Naithani et al., 2002]. Accordingly, $\tau_{y}(t)$ is assumed to be the actual wind stress (Figure 6.2a).

In order to evaluate the relative importance of the free and forced thermocline responses, the actual wind stress is split into two parts:

$$
\tau_{y}(t)=\tau_{s}(t)+\tau_{i}(t),
$$

where $\tau_{s}(t)$ is the seasonal wind stress (triggering the free oscillations), and $\tau_{i}(t)$ is the intraseasonal variability of the wind stress (causing the forced oscillations). The seasonal wind stress is made up of a constant wind stress $\tau_{c}$ during the dry season, and zero wind stress during the wet season, as shown in Figure 6.2b. It is thus defined by three parameters, the beginning and the duration of the dry season, and the value of the constant wind stress during the dry season $\tau_{c}$. The values of these parameters (given in Figure $6.2 \mathrm{~b}$ ) are determined by minimizing the integral of the least square difference between the functions $\tau_{y}(t)$ and $\tau_{s}(t)$.

\subsection{Thermocline response decomposition}

The downward displacement of the thermocline, $\xi(t, x, y)$, is defined as the thermocline response to the actual wind stress forcing $\tau_{y}(t)$. We call it the complete thermocline response, and it can be decomposed as follows:

$$
\xi(t, x, y)=\underbrace{\xi_{\text {sno }}(t, x, y)+\xi_{\text {so }}(t, x, y)}_{\xi_{s}(t, x, y)}+\xi_{i}(t, x, y)+\xi_{\text {syn }}(t, x, y),(6.6)
$$


where

- the seasonal response $\xi_{s}(t, x, y)$ is the thermocline response to the seasonal wind stress $\tau_{s}(t)$;

- the intraseasonal response $\xi_{i}(t, x, y)$ is the thermocline response to the intraseasonal variability of the wind stress $\tau_{i}(t)$;

- the synergistic term $\xi_{s y n}(t, x, y)$ is due to the non-linearity of the model [Stein and Alpert, 1993].

The seasonal response $\xi_{s}$ cannot be considered as purely oscillatory. Indeed, the thermocline oscillates about two equilibrium positions: a tilted thermocline (downward toward the north) during the dry season, and a horizontal one during the wet season, as is explained in Appendix A. Therefore the seasonal response $\xi_{s}$ is split into two parts:

- the reference non-oscillating part of the seasonal response $\xi_{\text {sno }}(t, x, y)$ is made up of two equilibrium states: it is the time-independent response to $\tau_{c}$ during the dry season, and it is zero during the wet season;

- the oscillating part of the seasonal response $\xi_{s o}(t, x, y)$ represents the oscillations of the seasonal response about these two equilibrium states.

The components $\xi, \xi_{s}$ and $\xi_{i}$ are computed by means of the reducedgravity model, using $\tau_{y}, \tau_{s}$ and $\tau_{i}$, respectively, as wind stress forcing. The dry season part of $\xi_{\text {sno }}$ is obtained by means of the reduced-gravity model using $\tau_{c}$ as wind stress, and its wet season part is zero. The other components $\xi_{\text {syn }}$ and $\xi_{\text {so }}$ are deduced from them since $\xi_{\text {syn }}=\xi-\xi_{s}-\xi_{i}$ and $\xi_{\text {so }}=\xi_{s}-\xi_{\text {sno }}$.

Only two of these contributions may be regarded as oscillations: $\xi_{\text {so }}$ and $\xi_{i}$ are now defined as the free and forced thermocline oscillations, respectively. For the Reader's convenience, $\xi_{\text {sno }}$ will be termed the non-oscillating seasonal response. The name and symbol of all the thermocline responses are gathered in Table 6.1. We now define several tools to analyze all these contributions.

\section{Width-average}

The thermocline responses can be averaged over the lake width:

$$
\bar{\xi}(t, y)=\frac{\int_{w_{1}(y)}^{w_{2}(y)} \xi(t, x, y) d x}{\int_{w_{1}(y)}^{w_{2}(y)} d x} .
$$




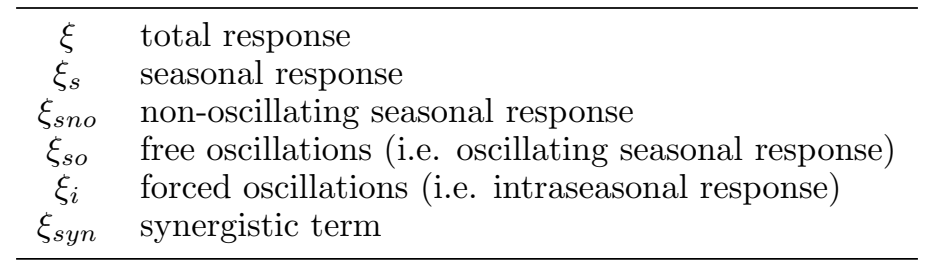

Table 6.1. Meaning of the symbols associated with the various components of the thermocline reponse to the wind forcing.

The lake width is determined by $w_{1}(y)$ on the left and $w_{2}(y)$ on the right (Figure 6.5). Figure 6.6 presents the width-averaged annual cycles of the complete thermocline response $\xi$ and its four contributions: the non-oscillating seasonal response $\xi_{\text {sno }}$, the free oscillations $\xi_{\text {so }}$, the forced oscillations $\xi_{i}$, and the synergistic term $\xi_{\text {syn }}$. The complete response is clearly oscillating, and the thermocline is more tilted downward toward the north during the dry season, which is why we have introduced $\xi_{\text {sno. }}$. The free oscillations seem to be only significant at the season changes. This is not the case with the forced oscillations that are present all year long, even if they are larger during the dry season. It has to be noted here that the relatively quick damping of the free oscillations is mainly due to the viscosity $\nu$, which is chosen to be equal to $3 \mathrm{~m}^{2} / \mathrm{s}$ here in order to be consistent with the former studies [Naithani et al., 2002, Naithani et al., 2003]. The synergistic term is not negligible and is also of an oscillating nature.

\section{Average over a specific region}

The thermocline responses can be averaged over a specific region $\Omega_{i}$ :

$$
\hat{\xi}(t)=\frac{\int_{\Omega_{i}} \xi(t, x, y) d x d y}{\int_{\Omega_{i}} d x d y} .
$$

We define a southern and a northern region (Figure 6.5). Figure 6.7 presents the mean annual cycles of four thermocline responses $\left(\xi, \xi_{\text {sno }}, \xi_{\text {so }}\right.$ and $\left.\xi_{i}\right)$ in these two regions. In the south (resp. in the north), all the oscillating responses present thirteen local minima (resp. maxima) per year, corresponding to an oscillation period of about 28 days, which is consistent with the above-mentioned three- to four-week oscillation period. Figure 6.7 also shows that the amplitude of the forced oscillations is much larger than that of the free ones (which are only significant at the season changes, as mentioned previously). Moreover the forced oscillations are more likely to fit the 


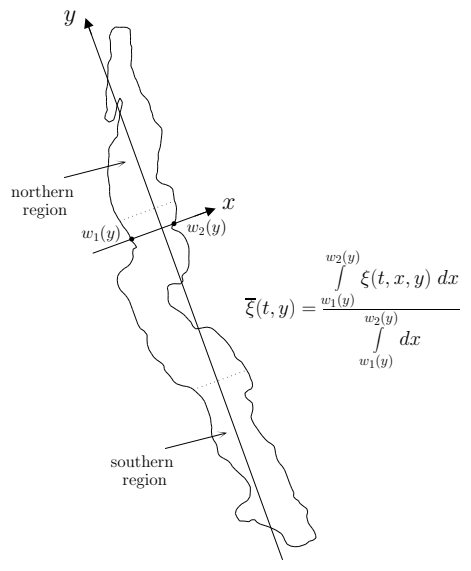

Fig. 6.5. A width-averaged response $\bar{\xi}(t, y)$ is computed over the lake width that depends on $y$. This width is determined by $w_{1}(y)$ on the left and $w_{2}(y)$ on the right. A response averaged over the southern and northern regions of the lake are averaged over the regions below a third and above the two thirds of the lake length, respectively.

complete response than the free ones. The complete thermocline response $\xi$ seems to be essentially made up of both the non-oscillating seasonal response $\xi_{\text {sno }}$ and the intraseasonal response $\xi_{i}$.

\section{Average over the whole lake - the concept of mean amplitude}

Since the surface layer volume is constant, the average of a thermocline response over the whole lake is zero. We then introduce the concept of mean amplitude that uses the absolute value of the thermocline responses. We define the mean amplitude of a thermocline response as follows:

$$
\tilde{\xi}(t)=\frac{\int_{\Omega}|\xi(t, x, y)| d x d y}{\int_{\Omega} d x d y},
$$

where $\Omega$ is the whole lake surface. The annual mean amplitude (i.e. $\tilde{\xi}$ averaged over the whole year) of the free and forced oscillations are equal to $7.87 \mathrm{~m}$ and $18.75 \mathrm{~m}$, respectively. The mean amplitude of the forced oscillations is thus, on average, more than twice as large as that of the free ones.

Figure 6.8 presents the evolution of the mean amplitude of the free and forced oscillations and their ratio. The forced oscillations are present all year round. Their mean amplitude is maximum during the dry season and then 


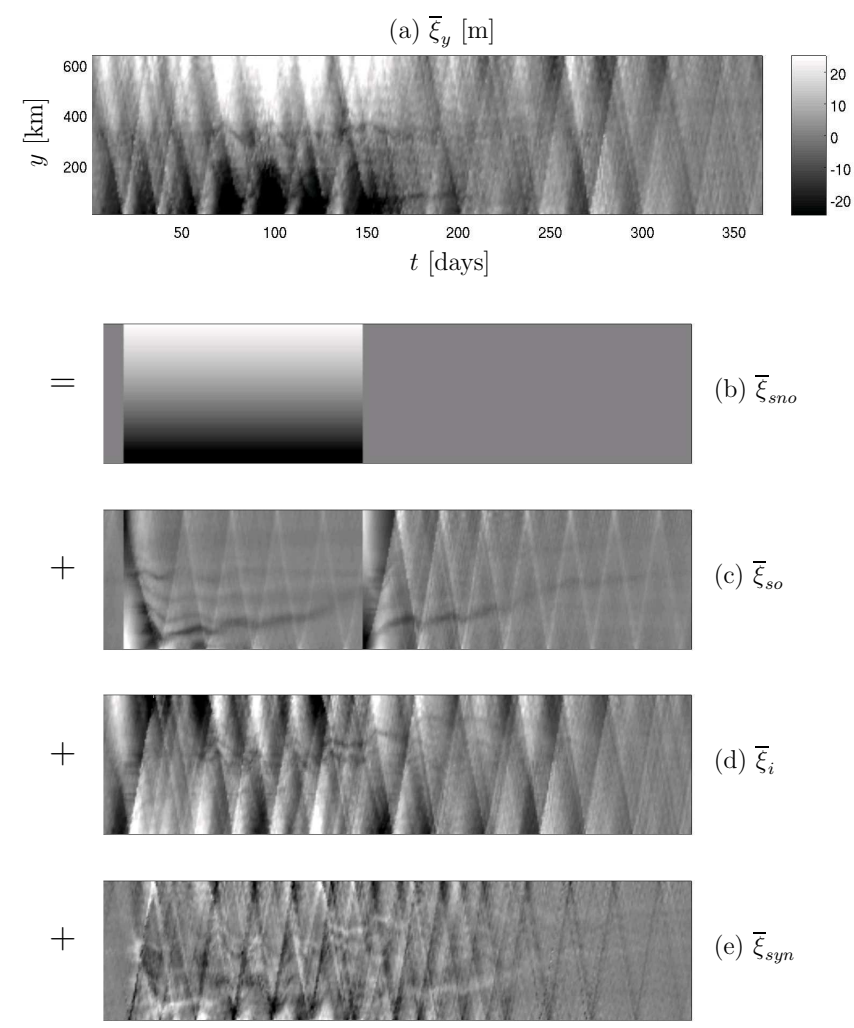

Fig. 6.6. Annual cycles of the width-averaged complete response (a), the width-averaged non-oscillating seasonal response (b), the width-averaged free oscillations (c), the width-averaged forced oscillations (d), and the width-averaged synergistic term (e).

decreases during the wet season. Their amplitude is always larger than that of the free oscillations, except at the season changes when the amplitude of the free oscillations strongly increases before decreasing exponentionally. Except at the season changes, the mean amplitude of the forced oscillations is over twice as large as that of the free oscillations, and even more so during the dry season (about five times on average).

The annual mean amplitude of the synergistic term is equal to $13.40 \mathrm{~m}$. Therefore, the synergistic term $\xi_{\text {syn }}$ is not negligible. This is partly due to the non-linearity of the reduced-gravity model equations. The wettingdrying algorithm, which prevents the thermocline from outcropping, also adds some non-linearity to the model. Moreover, the seasonal and intrasea- 

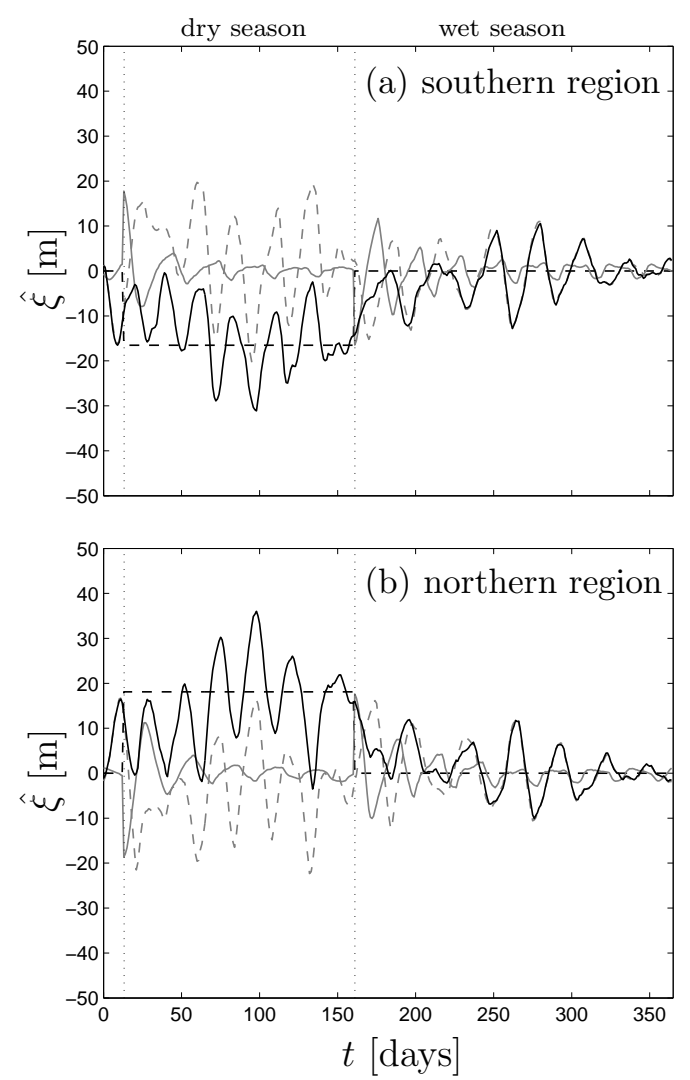

Fig. 6.7. Annual cycles of the complete response (solid black), the nonoscillating seasonal response (dashed black), the free oscillations (solid gray) and the forced oscillations (dashed gray), averaged over the southern (a) and northern (b) regions of the lake. These regions are defined as the ones below a third and above the two thirds of the lake length, respectively (Figure 6.5).

sonal responses are often out of phase, which is likely to increase the amplitude of the synergistic term.

\section{Selecting the most realistic reponse components}

Is the complete thermocline response mainly composed of the non-oscillating seasonal response $\xi_{\text {sno }}$, the free oscillations $\xi_{\text {so }}$, the forced oscillations $\xi_{i}$, or a combination of several of these three thermocline responses? There are seven possible combinations, composed of one, two or three terms taken among $\xi_{s n o}, \xi_{s o}$ and $\xi_{i}$. We call a signal the difference between the complete response $\xi$ and one of these combinations. That the mean amplitude of a 
(a)

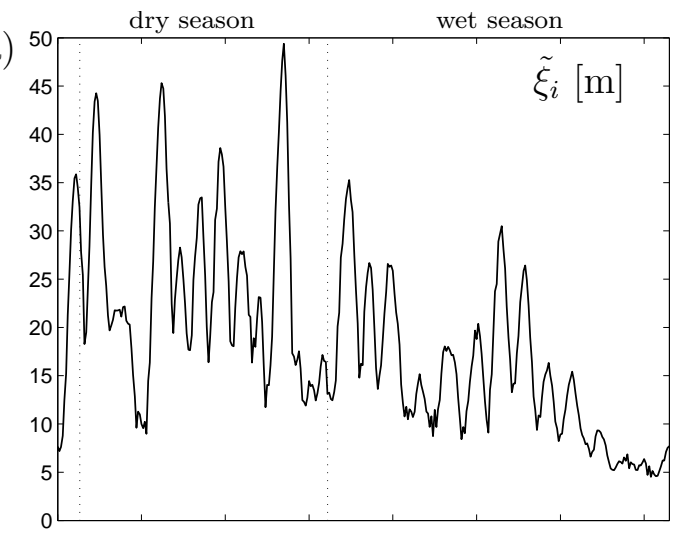

(b)

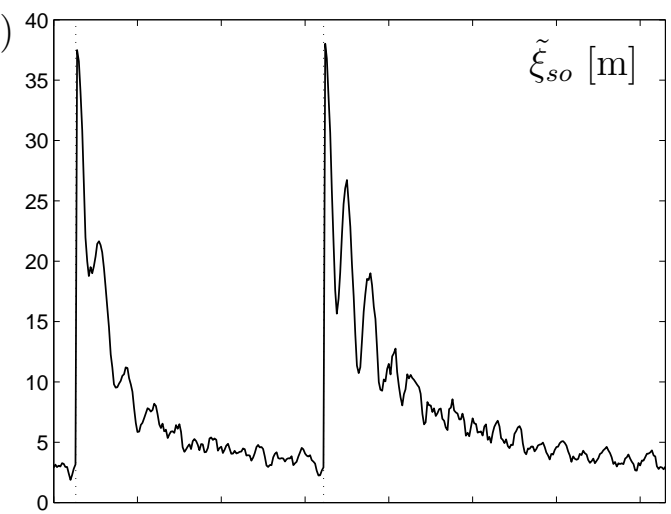

(c)

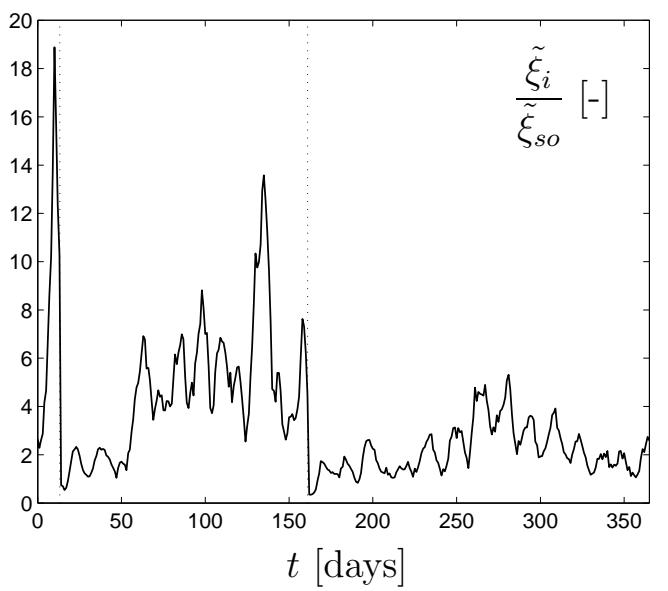

Fig. 6.8. Evolution of the mean amplitude [m] of the forced (a) and free (b) oscillations, and evolution of their ratio [-] (c). 
given signal is small indicates that the associated combination is a good approximation of the complete response. Table 6.2 presents the mean amplitude of the signals related to the seven possible combinations. It shows that the four combinations containing $\xi_{\text {sno }}$ are the best ones. So the nonoscillating seasonal response is essential to describe the complete response. According to this method, the best combination is $\xi_{\text {sno }}+\xi_{i}$. The free oscillations $\xi_{\text {so }}$ are not only useless to describe the complete response, but the approximation to the complete response gets worse when $\xi_{s o}$ is added.

\begin{tabular}{cc}
\hline signal & amplitude \\
\hline$\xi-\left(\xi_{\text {sno }}+\xi_{i}\right)$ & $13.12 \mathrm{~m}$ \\
$\xi-\left(\xi_{\text {sno }}+\xi_{\text {so }}+\xi_{i}\right)$ & $13.4 \mathrm{~m}$ \\
$\xi-\xi_{\text {sno }}$ & $16.75 \mathrm{~m}$ \\
$\xi-\left(\xi_{\text {sno }}+\xi_{\text {so }}\right)$ & $18.39 \mathrm{~m}$ \\
$\xi-\xi_{i}$ & $22.74 \mathrm{~m}$ \\
$\xi-\xi_{\text {so }}$ & $23.27 \mathrm{~m}$ \\
$\xi-\left(\xi_{\text {so }}+\xi_{i}\right)$ & $23.43 \mathrm{~m}$ \\
\hline
\end{tabular}

Table 6.2. Mean amplitude of the different signals composed of the difference between $\xi$ and the various combinations of $\xi_{\text {sno }}, \xi_{\text {so }}$ and $\xi_{i}$.

\subsection{Conclusion}

A hydrodynamic model was built that reproduces rather well the results of former simulations of the thermocline oscillations in Lake Tanganyika. Former studies established the presence of free oscillations with a period of three to four weeks [Naithani et al., 2003], and noted that the thermocline oscillations are also due to forced oscillations with the same three- to fourweek period [Naithani et al., 2002] (the period of theses forced oscillations are due to the intraseasonal variability of the wind stress in the region of the lake). But no comparison between the two oscillation types had been achieved so far. We showed that, in Lake Tanganyika, the forced oscillations of the thermocline are significantly larger than their free counterparts: their mean annual amplitude is more than twice as large. It was also seen that combining the non-oscillating seasonal response of the thermocline (which is tilted downward toward the north during the dry season and horizontal for the rest of the year) and the forced oscillations yields a response that is closer to the complete one than any other response combination. 


\section{Appendix A Seasonal response decomposition}

As illustrated by Figure 6.2b, the seasonal component of the surface wind stress is defined to be

$$
\tau_{s}(t)=\left\{\begin{array}{cc}
\tau_{c} & \text { during the dry season } \\
0 & \text { during the wet season }
\end{array},\right.
$$

where $\tau_{c}$ is a positive constant. The response of the thermocline to this wind forcing, i.e. the seasonal thermocline response $\xi_{s}$, is made up of an oscillating part $\xi_{s o}$ and a non-oscillating one $\xi_{\text {sno. }}$. The latter is the steady-state response to the seasonal wind stress. Accordingly, it obeys the momentum equation

$$
\epsilon g\left(h+\xi_{\text {sno }}\right) \frac{\partial \xi_{\text {sno }}}{\partial y}=\frac{\tau_{s}}{\rho},
$$

Then, it is readily seen that the seasonal non-oscillating thermocline displacement is zero during the wet season (i.e. the thermocline is horizontal) and is equal to the following expression during the dry season

$$
\xi_{\text {sno }}(t, x, y)=-h+\sqrt{\frac{2 \tau_{c}(y+Y)}{\epsilon g \rho}},
$$

where $Y$ is a constant whose value must guarantee that the volume of the surface layer remains time-independent. In other words, this constant is such that the integral of $\xi_{\text {sno }}$ over the surface of the lake $\Omega$ is zero, implying that $Y$ must satisfy

$$
\int_{\Omega} h d x d y=\int_{\Omega} \sqrt{\frac{2 \tau_{c}(y+Y)}{\epsilon g \rho}} d x d y .
$$

\section{Acknowledgements}

Eric Deleersnijder and Laurent White are a Research Associate and a honorary Postdoctoral Researcher, respectively, with the Belgian National Fund for Scientic Research (FNRS). The present study was carried out within the scope of the project "A second-generation model of the ocean system", which is funded by the Communauté Française de Belgique, as Actions de Recherche Concertées, under contract ARC 04/09-316. This work is a contribution to the construction of SLIM, the Second-generation Louvain-la-Neuve Ice-ocean Model (http://www.climate.be/SLIM). The authors are indebted to Jaya Naithani and Pierre-Denis Plisnier for useful discussions. Finally, the present study is linked to the CLIMFISH project (Climate change impact on the sustainable use of Lake Tanganyika fisheries), funded by the STEREO programme of Belgian Federal Science Policy and 
the framework agreement between DGCD (Belgian Development Cooperation) and the Royal Museum for Central Africa (RMCA), Tervuren, Belgium.

\section{Bibliography}

[Antenucci, 2005] Antenucci, J. P. (2005). Comment on "Are there internal Kelvin waves in Lake Tanganyika?". Geophysical Research Letters, 32(22). L22601 doi : 10.1029/2005GL024403.

[Antenucci and Imberger, 2001] Antenucci, J. P. and Imberger, J. (2001). Energetics of long internal gravity waves in large lakes. Limnology and Oceanography, 46(7):1760-1773.

[Balzano, 1998] Balzano, A. (1998). Evaluation of methods for numerical simulation of wetting and drying in shallow water flow models. Coastal Engineering, 34(12):83-107.

[Coulter and Spigel, 1991] Coulter, G. W. and Spigel, R. H. (1991). Hydrodynamics. In Coulter, G. W., editor, Lake Tanganyika and its life, pages 49-75. Oxford University Press, New York, USA.

[Csanady, 1967] Csanady, G. T. (1967). Large-scale motion in the great lakes. Journal of Geophysical Research, 72(16):4151-4162.

[Descy et al., 2005] Descy, J.-P., Hardy, M.-A., Sténuite, S., Pirlot, S., Leporcq, B., Kimirei, I., Sekadende, B., Mwaitega, S. R., and Sinyenza, D. (2005). Phytoplankton pigments and community composition in Lake Tanganyika. Freshwater Biology, 50(4):668-684.

[Goswami and Shukla, 1984] Goswami, B. N. and Shukla, J. (1984). Quasi-periodic oscillations in a symmetric general circulation model. Journal of the Atmospheric Sciences, 41(1):20-37.

[Gourgue et al., 2007] Gourgue, O., Deleersnijder, and White, L. (2007). Toward a generic method for studying water renewal, with application to the epilimnion of Lake Tanganyika. Estuarine, Coastal and Shelf Science. In press, doi:10.1016/j.ecss.2007.05.009.

[Hanert et al., 2005] Hanert, E., Le Roux, D. Y., Legat, V., and Deleersnijder, E. (2005). An efficient Eulerian finite element method for the shallow water equations. Ocean Modelling, 10(1-2):115-136.

[Kanari, 1975] Kanari, S. (1975). The long-period internal waves in Lake Biwa. Limnology and Oceanography, 20(4):544-553.

[Kielmann and Simons, 1984] Kielmann, J. and Simons, T. J. (1984). Some aspects of baroclinic circulation models. In Hutter, K., editor, Hydrodynamics of Lakes: CISM Lectures, pages 235-285. Springer Verlag Wien New York, Vienna, Austria.

[Krishnamurti and Bhalme, 1976] Krishnamurti, T. N. and Bhalme, H. N. (1976). Oscillations of a Monsoon system. Part I. Observational aspects. Journal of the Atmospheric Sciences, 33(10):1937-1954.

[Lin et al., 2000] Lin, J. W.-B., Neelin, J. D., and Zeng, N. (2000). Maintenance of tropical intraseasonal variability: impact of evaporation-wind feedback and midlatitude storms. Journal of the Atmospheric Sciences, 57(17):2793-2823.

[Madden and Julian, 1971] Madden, R. A. and Julian, P. R. (1971). Detection of 40-50 day oscillation in the zonal wind in the Tropical Pacific. Journal of the Atmospheric Sciences, 28(5):702-708. 
[Madden and Julian, 1994] Madden, R. A. and Julian, P. R. (1994). Observations of the 40-50-day tropical oscillation - A review. Monthly Weather Review, $122(5): 814-837$.

[Marchal, 2005] Marchal, E. (2005). Simulation par lments finis des oscillations induites par le vent de la thermocline dans le Lac Tanganyika. Master's thesis, Universit catholique de Louvain, Louvain-la-Neuve, Belgium.

[Mortimer, 1974] Mortimer, C. H. (1974). Lake kydrodynamics. Mitteilungen Internationale Vereinigung für Theoretische und Angewandte Limnologie, 20:124197.

[Naithani and Deleersnijder, 2004] Naithani, J. and Deleersnijder, E. (2004). Are there internal Kelvin waves in Lake Tanganyika? Geophysical Research Letters, 31(6). L06303, doi:10.1029/2003GL019156.

[Naithani et al., 2002] Naithani, J., Deleersnijder, E., and Plisnier, P.-D. (2002). Origin of intraseasonal variability in Lake Tanganyika. Geophysical Research Letters, 29(23). 2093, doi:10.1029/2002GL015843.

[Naithani et al., 2003] Naithani, J., Deleersnijder, E., and Plisnier, P.-D. (2003). Analysis of wind-induced thermocline oscillations of Lake Tanganyika. Environmental Fluid Mechanics, 3(1):23-39.

[Neelin et al., 1987] Neelin, J. D., Held, I. M., and Cook, K. H. (1987). Evaporationwind feedback and low-frequency variability in the tropical atmosphere. Journal of the Atmospheric Sciences, 44(16):2341-2348.

[O'Reilly et al., 2003] O'Reilly, C. M., Alin, S. R., Plisnier, P.-D., Cohen, A. S., and McKee, B. A. (2003). Climate change decreases aquatic ecosytem productivity of Lake Tanganyika, Africa. Nature, 424:766-768.

[Plisnier et al., 1999] Plisnier, P.-D., Chitamwebwa, D., Mwape, L., Tshibangu, K., Langenberg, V., and Coenen, E. (1999). Limnological annual cycle inferred from physical-chemical fluctuations at three stations of Lake Tanganyika. Hydrobiologia, 407:45-58.

[Schwab, 1977] Schwab, D. J. (1977). Internal free oscillations in Lake Ontario. Limnology and Oceanography, 22(4):700-708.

[Stein and Alpert, 1993] Stein, U. and Alpert, P. (1993). Factor separation in numerical simulations. Journal of the Atmospheric Sciences, 50(14):2107-2115. 